\title{
Single Palmar Crease
}

National Cancer Institute

\section{Source}

National Cancer Institute. Single Palmar Crease. NCI Thesaurus. Code C87120.

A single transverse palmar crease is found in $5 \%$ of newborns and is frequently inherited as a familial trait. However, single palmar creases can be associated with Down's syndrome and other genetic disorders, or with fetal alcohol syndrome. 